\title{
Making decisions in the dark basement of the brain: A look back at the GPR model of action selection and the basal ganglia
}

\author{
Mark D. Humphries $^{1}$ (D) $\cdot$ Kevin Gurney ${ }^{2}$ \\ Received: 29 April 2021 / Accepted: 6 July 2021 / Published online: 17 July 2021 \\ (c) The Author(s), under exclusive licence to Springer-Verlag GmbH Germany, part of Springer Nature 2021
}

\begin{abstract}
How does your brain decide what you will do next? Over the past few decades compelling evidence has emerged that the basal ganglia, a collection of nuclei in the fore- and mid-brain of all vertebrates, are vital to action selection. Gurney, Prescott, and Redgrave published an influential computational account of this idea in Biological Cybernetics in 2001. Here we take a look back at this pair of papers, outlining the "GPR" model contained therein, the context of that model's development, and the influence it has had over the past twenty years. Tracing its lineage into models and theories still emerging now, we are encouraged that the GPR model is that rare thing, a computational model of a brain circuit whose advances were directly built on by others.
\end{abstract}

Keywords Striatum $\cdot$ Motor programs $\cdot$ Movement selection $\cdot$ Decision making $\cdot$ Disinhibition $\cdot$ Direct and indirect pathways

\section{Introduction}

On the first day of my $\mathrm{PhD}$ in October 1998, I (MDH) was handed a thick, 51-page report, held between covers of pale blue card on which were printed the ominous words "Analysis and simulation of a model of the basal ganglia," and instructed by my supervisor, one Kevin Gurney, to read all the contents therein. Intimidating as it was for a first year $\mathrm{PhD}$ student fresh from their undergraduate studies, this behemoth of a technical report would become the foundation of a pair of classic papers published together in Biological Cybernetics in 2001, both with the same author line of Gurney, Prescott and Redgrave: "A computational model of action selection

Communicated by Benjamin Lindner and Paul Tiesinga.

To highlight the scientific impact of our Journal over the last decades, we asked authors of highly influential papers to reflect on the history of their study, the long-term effect it had, and future perspectives of their research. We trust the reader will enjoy these first-person accounts of the history of big ideas in Biological Cybernetics.

$凶$ Mark D. Humphries

mark.humphries@nottingham.ac.uk

Kevin Gurney

k.gurney@sheffield.ac.uk

1 University of Nottingham, Nottingham, UK

2 University of Sheffield, Sheffield, UK in the basal ganglia I: A new functional anatomy" (Gurney et al. 2001a) and "A computational model of action selection in the basal ganglia II: Analysis and simulation of behaviour" (Gurney et al. 2001b).

Here we take a look back at this pair of papers, at their context, their influence, and what the future may hold for the model of the brain they contain. In reference to their author line, and in keeping with the common name they have acquired over the last 20 years, we refer to the model as the GPR model throughout.

\section{Why the basal ganglia at all?}

The basal ganglia comprise the massive striatum, sitting underneath the cortex in much of the forebrain, and a group of much smaller deep-lying nuclei (Fig. 1). Their functional role has long perplexed us: Kinnear-Wilson called them the dark basement of the brain as far back as the 1920s (Graybiel 2000), a feeling still evoked in many of its researchers now, not least because they are seemingly involved in so many disorders. It is from the striatum that dopamine is lost when midbrain dopaminergic neurons die in Parkinson's disease, leading to the classic clinical signs of akinesia, rigidity, and tremor. The death of the principle, projection neurons of the striatum leads the appearance of chorea, the unpredictable, uncontrolled limb movements in Huntington's disease. So 


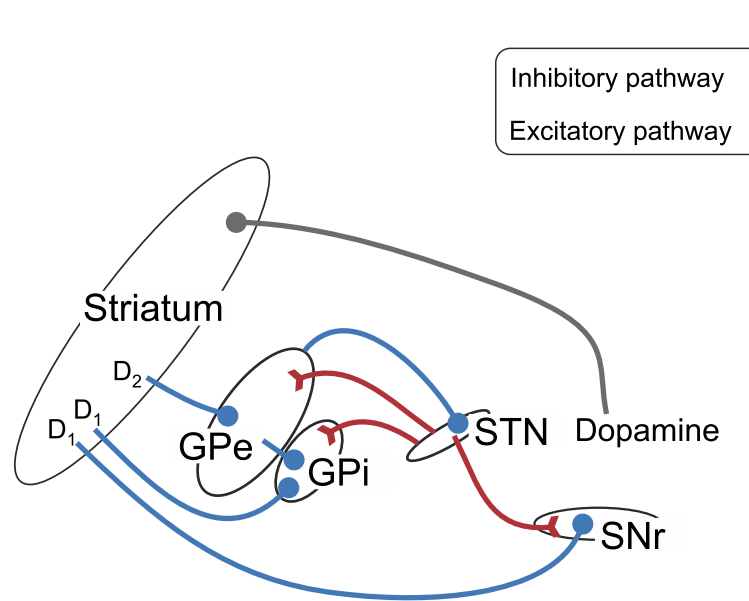

(a)

Fig. 1 Anatomy of the basal ganglia. (a) Nuclei of the basal ganglia nuclei and their internal connections. Nuclei positions and sizes correspond to their approximate relative locations and sizes in a sagittal section of the rodent brain. Dopamine is released into the striatum by inputs from the midbrain, and striatal neurons express either the D1 or D2 type of dopamine receptor. (b) External connections of the basal

it had long been thought that, whatever they do, it involved movement in some way (Denny-Brown 1962).

By the late 1990s, a consensus was emerging around the idea that the basal ganglia are crucial to the selection of movements, but not their initiation (Mink and Thach 1993; Marsden and Obeso 1994; Mink 1996). This idea beautifully explained how the basal ganglia could be seemingly so central to such a wide range of movement disorders, how stimulating neurons within them could immediately evoke movement, and yet damage to the basal ganglia did not prevent movement from happening. What it left open was how: how exactly did the basal ganglia select movements?

At the time, sketched ideas for this centred on the concept of disinhibition (Deniau and Chevalier 1985; Chevalier and Deniau 1990). Neurons of the basal ganglia's output nuclei are persistently active, at around 60 spikes per second in primates, and GABAergic, so constantly inhibiting every neuron they target. The disinhibition concept proposed that turning off this persistently inhibitory output signals the selection of the motor program encoded by the target neurons.

At about the same time, there emerged influential conceptual models of the wiring between these nuclei. One model proposed two pathways from the striatum that converged on the output nuclei of the basal ganglia. The striatal neurons of the so-called direct pathway sent their axons directly to the neurons of the output nuclei; the striatal neurons of the "indirect" pathway sent their axons to the globus pallidus pars externa (GPe in Fig 1), whose neurons in turn ganglia to the rest of the brain. Rectangular boxes indicate regions outside the basal ganglia. Neurons of the globus pallidus pars interna (GPi) and substantia nigra pars reticulata $(\mathrm{SNr})$ receive similar inputs and are the main source of projections outside of the basal ganglia, and so are collectively termed the "output nuclei" here. GPe: globus pallidus pars externa; STN: subthalamic nucleus

project to the output nuclei (Albin et al. 1989; Alexander and Crutcher 1990; DeLong 1990). Another model proposed that the basal ganglia were organised topographically, so connections between nuclei formed parallel loops (Alexander et al. 1986; Alexander and Crutcher 1990). Moreover, it was becoming clear that connections from the tiny subthalamic nucleus, the only source of excitatory glutamate within the basal ganglia, to the output nuclei were a further key player (Parent and Hazrati 1995). Unclear was how to link these conceptual models of wiring to the conceptual models of selection.

Into this milieu were launched what was intended to be a triptych of papers. The first, bylined (Redgrave et al. 1999), argued how the idea of movement selection can be understood as a special case of the formal problem of action selection and showed how the basal ganglia were seemingly ideally placed to solve that problem. Not least that these parallel loops were, at a fine scale, the substrate for representing competing actions. The second, bylined (Prescott et al. 1999) put this hypothesis into the context of the rest of the brain, arguing that the brain contains many-layered control of movement, stretching from the deepest brainstem to the cortex, and the basal ganglia has privileged access to many of these layers, positioning it as a key locus for controlling actions. Together, these papers made a compelling case for a more nuanced account for what the basal ganglia do: they select actions. 
The third planned paper, which transpired to be the pair of papers that are our subject here, was the how: the model that synthesised all the above conceptual ideas into a single quantitative computational account of how the basal ganglia implement action selection. And it answered two crucial questions missing from all the words and the box-and-arrow diagrams (Albin 2001): How does it resolve competition between actions; and how does it switch actions?

\section{The GPR model}

The first paper (Gurney et al. 2001a) tackled the job of synthesising the above ideas and data into a single coherent whole, into the circuit for selection defined by the wiring of the basal ganglia; it also did the important job of formally defining what selection means. Figure 2 shows the resulting "functional anatomy" of the basal ganglia, so-called because it is anatomy read in the light of a functional hypothesis. This formalised how actions could be represented within the basal ganglia by parallel groups of neurons - "channels" in the paper's parlance. In this scheme, each channel through the basal ganglia represents an action, and the neurons' activity within a channel represents the salience of that action. The action with the highest salience ought to win the competition between the currently available actions, and be selected. Selection was defined using the concept of disinhibition: the selected channel of the output nucleus was the one whose activity sufficiently reduced for its inhibitory influence to be removed.

In the second paper (Gurney et al. 2001b) was laid out the formal mathematical model built on that anatomy, its analytical solutions, and simulations that illustrated their key points. The model was constructed at a population level, each channel within a given nucleus modelled as the ensemble activity of its neurons. A key element of the model was the modulation by dopamine of the striatal neuron's activity, building on recent data showing striatal neurons with dopamine D1 receptors formed the direct pathway and those with D2 receptors the indirect pathway. Critically, the paper synthesised data that suggested activating these receptors has opposite effects on neural activity, with D1 activation enhancing activity and D2 activation depressing activity.

This model of dopamine has proved remarkably resilient, and remains one of the key contributions of the GPR model. Of the further immediate contributions of this pair of papers, we highlight three.

First is that the new functional anatomy provided an influential bauplan for theories of the basal ganglia. As we discuss below, this template has formed the basis for numerous models of the basal ganglia from us and others, and brought into sharp focus mysteries of the basal ganglia, such as the func- tion of the striatum's internal wiring, some of which have yet to be resolved.

Second is the explanation for how the basal ganglia resolves the competition between its inputs. As laid out in the functional anatomy, the output nuclei receive an odd combination of focused inhibition from striatum and putative diffuse excitation from the subthalamic nucleus (Fig. 2a). By combining the channel idea with this anatomy, the paper proposed the basal ganglia act as an off-centre, on-surround network: competition between competing actions is realised by the balance of the focussed inhibition and broad excitation received by the neurons of each channel in the output nuclei. The winning channel — and hence action — would thus be the one whose balance of inputs tipped most strongly in favour of the inhibition and so had the most reduced activity.

This mechanism also explains how actions are switched between. When a more salient action becomes available, the model showed that the same off-centre, on-surround design naturally handles this: the new input further increases the output of the subthalamic nucleus, in turn increasing the activity of all the output channels, thus cancelling selection of the current action, while at the same time the corresponding new, stronger inhibitory signal from striatum inhibits its target population in the output nuclei.

A third contribution is the idea of capacity scaling, that the basal ganglia are able to automatically scale the activity of the output nuclei to cope with the number of competing actions. The problem facing the basal ganglia is that the subthalamic nucleus output is both excitatory and diffuse, so increasing its total input will increase activity across the output nuclei (Fig. 2a). Consequently, the more actions there are competing, the more input the subthalamic nucleus receives, the harder it is to select a single action by turning off one channel of the output nucleus.

But as shown in Fig. 2b, the subthalamic nucleus and globus pallidus form a negative feedback loop. Analysis in the second paper (Gurney et al. 2001b) showed that this was sufficient to automatically scale the activity in the subthalamic nucleus: the higher it is driven by its inputs, the more strongly it drives activity in the globus pallidus, which in turn inhibits activity in the subthalamic nucleus. Which all means that the subthalamic nucleus activity is automatically scaled down as the number of its inputs increases. Consequently, the activity of the output nuclei does not grow towards saturation as the number of competing actions increases-their capacity is scaled. No matter how many extra active inputs join the competition for selection, this feedback loop guarantees that the output activity will remain responsive to its striatal input, and thus allow selection to continue. 


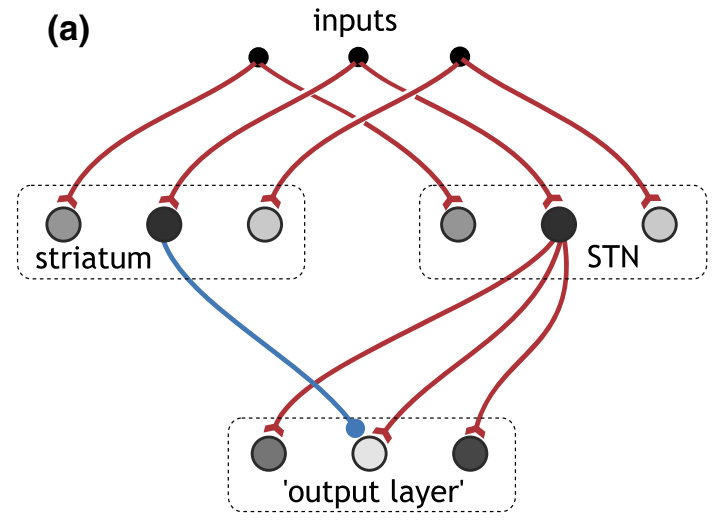

Fig. 2 Functional anatomy of the basal ganglia. As laid out in Gurney et al. (2001a). (a) The anatomy of how selection works. Populations or "channels" in each nucleus represent actions (circles). In this reading of the anatomy, populations of D1-expressing striatal neurons send inhibitory projections to their corresponding populations in the output nuclei; whereas populations in the subthalamic nucleus (STN) send diffuse excitatory projections to all populations in the output nuclei (we show only full connections of the central channel for clarity). Grey-scale

\section{Influence of the model}

The most immediate influence of the GPR model was the fecund research programme it sparked in our group (Gurney et al. 2004; Redgrave and Costa 2021). We embedded the model in the wider thalamocortical loop within which the basal ganglia sit, and explored how that influenced its capacity for action selection (Humphries and Gurney 2002). We created a large-scale spiking neuron version, now with hundreds of individual neurons in each nucleus, to show that the action selection hypothesis was compatible with both the detailed dynamics of individual nuclei and with a wide range of electrophysiological data (Humphries et al. 2006). With the same spiking model, we went on to show how deep brain stimulation of the subthalamic nucleus, an effective treatment for the cardinal motor signs of Parkinson's disease, can elicit a mixture of excitatory and inhibitory responses in the output nuclei (Humphries and Gurney 2012). Embodying the model in a mobile robot showed how smooth behavioral sequences naturally emerge from the changing saliences of each action-but also revealed where our understanding of action selection was lacking (Prescott et al. 2006). In a comprehensive review of the ventral basal ganglia, the part that has the nucleus accumbens as its input structure, we showed that the circuit originating from the core of the accumbens also conformed to the GPR model, thus casting its known roles in navigation and learning as a function of selecting appropriate responses (Humphries and Prescott 2010; Khamassi and Humphries 2012). We even subjected the poor thing to the Stroop task (Stafford and Gurney 2004, 2007).

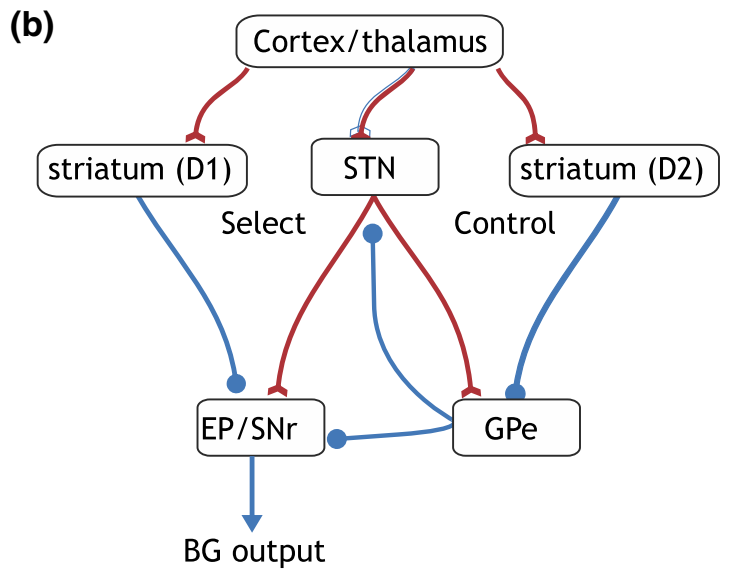

fills indicate an example of relative levels of activity in each "channel", with dark-to-light encoding high-to-low activity. In this example, the combination of focussed inhibition and diffuse excitation arriving at the output layer results in the central channel having little activity and the others more activity, indicating clean selection of the central channel's action. (b) Full functional anatomy, with interpretations of the functional role of the anatomically defined pathways

This body of work culminated in a complete theory of how the plasticity of the connections from the cortex to the striatum, controlled by dopamine signals that convey prediction errors, drives the learning and extinction of action selection via the basal ganglia (Gurney et al. 2015).

The GPR model found an immediate home in the work of Agnes Guillot and her colleagues, as part of the Psikharpax project to construct an artificial rat. Again embodied in a variety of mobile robots, Guillot's team explored its ability to control foraging (Girard et al. 2003), integrate its action selection with models for navigation (Girard et al. 2005), and be the "Actor" in Actor-Critic models of reinforcement learning (Khamassi et al. 2005). Benoit Girard has continued this vein of work into new areas, including a dynamical systems analysis of the model (Girard et al. 2008), and a major piece of work to establish a version that more fully captured the basal ganglia circuit in primates (Liénard and Girard 2014) - a full spiking neuron version of that primate model has recently followed (Girard et al. 2020).

A version of the GPR model of the basal ganglia also sits at the heart of SPAUN (Eliasmith et al. 2012), Chris Eliasmith and colleagues' 2.5 million spiking neuron model of a brain architecture for cognitive tasks. Naturally, this basal ganglia model handles the action selection process in all tasks SPAUN is trained to do. Critical to its place in SPAUN though is their addition of plasticity between the cortical-striatal connections (Stewart et al. 2012), essential for the model to relearn associations between the states of the world and the appropriate actions in them for each new task SPAUN faces. 
Rafal Bogacz took the GPR model in a different direction. He noticed that the functional architecture laid out in the first paper (Gurney et al. 2001a) could potentially implement the multi-sequential probability ratio test (the MSPRT), a Bayesian algorithm for making a decision based on the accumulating evidence for multiple options. In this version of the model, each channel represents an option, the input to striatum represents the momentary evidence to be accumulated, and the basal ganglia output at each moment in time represents the (negative log) of the conditional probability of each option given the evidence so far-the negative log so that the decision is made when one of the outputs falls below a threshold, again making use of the disinhibition concept. The resulting paper (Bogacz and Gurney 2007), co-authored with one of us (KG), was perhaps the first to draw formal links between the decision making literature and action selection ideas. The role of the basal ganglia in decision-making is now a burgeoning research field, with deep development of the theory that the basal ganglia implement an optimal algorithm for decision-making (Zhang and Bogacz 2010; Lepora and Gurney 2012; Bogacz et al. 2016; Caballero et al. 2018), combined with compelling experimental evidence that the striatum plays a key, causal role in decision making (Ding and Gold 2010, 2012; Yartsev et al. 2018).

The GPR model's lineage can be traced to many recent models that have used its ideas as a springboard for a deep exploration of the basal ganglia. These include Fountas and Shanahan's (2017) spiking model that explored how oscillations in the input to the basal ganglia altered their consequent output, Dunovan and colleagues' (Dunovan et al. 2019) spiking model that linked the dynamics of the basal ganglia to the behavioural parameters of decision making, including that the rate of evidence accumulation was defined by the difference in activity between channels of the "direct" pathway, and Lindahl and Kotaleski's large-scale spiking model (Lindahl and Kotaleski 2016) that exhaustively explored what the connections within the basal ganglia contributed to both their dynamical repertoire and their ability to perform action selection. And showing it's still going strong nearly 20 years on, Gilbertson and Steele made use of a variant of the GPR model in their proposal that the combination of dopamine's immediate and long-term effects in the striatum can allow the basal ganglia to optimally solve the exploration-exploitation trade-off in action selection (Gilbertson and Steele 2020).

\section{The future}

Any good model of a specific neural circuit also sets out a research programme for the future, by showing what we don't know, and what we need to know. One such unknown was the contribution of the internal circuitry of the striatum. At the time, we knew of three interneuron types (Kawaguchi
1993), and of the anatomical evidence for connections made between the projection neurons of the striatum (Wilson and Groves 1980), but little else; now the number of interneurons types has at least doubled, and we know the microcircuit of the striatum in some detail from both anatomical and electrophysiological data (Tepper et al. 2018). But as the GPR model illustrated, the basal ganglia did not need this striatal microcircuit to perform action selection-so what was it for? This gap in our knowledge has driven a programme of computational work on the striatum's microcircuit by us (Humphries et al. 2009, 2010; Tomkins et al. 2014) and others (Yim et al. 2011; Spreizer et al. 2017), culminating in a full-scale model of biophysical striatum from HellgrenKotaleski and colleagues (Hjorth et al. 2020).

Our knowledge of the basal ganglia is ever-evolving. In a further example, whereas the globus pallidus is treated as a single entity in the GPR model, we now know it contains at least two distinct populations, the proto-pallidal and arkypallidal, with different inputs, targets, and dynamics (for review see Gittis et al. 2014). Moreover, the feedback connections from the globus pallidus to striatum, only hinted at by data available during the model's development in the late 90s (Walker et al. 1989; Rajakumar et al. 1994), are now a well-studied pathway that originate from the arkypallidal population (Bevan et al. 1998; Mallet et al. 2012; Corbit et al. 2016). The venerable GPR model has been updated to integrate these new data too (Suryanarayana et al. 2019).

But as our knowledge of the basal ganglia continues to evolve, the reader may have the nagging question of whether it is time to finally abandon the GPR model, to move on to a new view of how the basal ganglia function. And even if we do not make any explicit decision to abandon a model, we are still left with the computational equivalent of the Ship of Theseus paradox: that if we keep updating and altering the model with each new advance in our knowledge of the basal ganglia brought by experimental data, then what remains of the original?

Perhaps that is the wrong view of what a model is for. Rather, like all good models, we know the GPR model was flawed at the outset: its test was not whether it was 'right', but whether it was useful. And as we hope we have demonstrated here, the GPR model has been decidedly useful to us and many others over the 20 years since its publication, and so shall exist in some form for as long as that may continue.

Acknowledgements We thank Peter Redgrave and Tony Prescott for a reading a draft of this retrospective.

\section{Declarations}

Conflicts of interest The authors declare that they have no conflict of interest. 


\section{References}

Albin RL (2001) End of lines and boxes. Mov Disord 16:405-406

Albin RL, Young AB, Penney JB (1989) The functional anatomy of basal ganglia disorders. Trends Neurosci 12:366-375

Alexander GE, Crutcher MD (1990) Functional architecture of basal ganglia circuits: neural substrates of parallel processing. Trends Neurosci 13:266-272

Alexander GE, DeLong MR, Strick PL (1986) Parallel organization of functionally segregated circuits linking basal ganglia and cortex. Annu Rev Neurosci 9:357-381

Bevan MD, Booth PAC, Eaton SA, Bolam JP (1998) Selective innervation of neostriatal interneurons by a subclass of neuron in the globus pallidus of the rat. J Neurosci 18(22):9438-9452

Bogacz R, Gurney K (2007) The basal ganglia and cortex implement optimal decision making between alternative actions. Neural Comput 19(2):442-477

Bogacz R, Martin Moraud E, Abdi A, Magill PJ, Baufreton J (2016) Properties of neurons in external globus pallidus can support optimal action selection. PLoS Comput Biol 12(7):e1005004

Caballero JA, Humphries MD, Gurney KN (2018) A probabilistic, distributed, recursive mechanism for decision-making in the brain. PLoS Comput Biol 14:e1006033

Chevalier G, Deniau JM (1990) Disinhibition as a basic process in the expression of striatal function. Trends in Neurosci 13:277-280

Corbit VL, Whalen TC, Zitelli KT, Crilly SY, Rubin JE, Gittis AH (2016) Pallidostriatal projections promote $\beta$ oscillations in a dopamine-depleted biophysical network model. J Neurosci 36:5556-5571

DeLong MR (1990) Primate models of movement disorders of basal ganglia origin. Trends Neurosci 13(7):281-285

Deniau JM, Chevalier G (1985) Disinhibition as a basic process in the expression of striatal functions. II. The striato-nigral influence on thalamocortical cells of the ventromedial thalamic nucleus. Brain Res 334(2):227-233

Denny-Brown D (1962) The basal ganglia and their relation to disorders of movement. OUP, Oxford

Ding L, Gold JI (2010) Caudate encodes multiple computations for perceptual decisions. J Neurosci 30(47):15747-15759

Ding L, Gold JI (2012) Separate, causal roles of the caudate in saccadic choice and execution in a perceptual decision task. Neuron 75(5):865-874

Dunovan K, Vich C, Clapp M, Verstynen T, Rubin J (2019) Rewarddriven changes in striatal pathway competition shape evidence evaluation in decision-making. PLoS Comput Biol 15:e1006998

Eliasmith C, Stewart TC, Choo X, Bekolay T, DeWolf T, Tang Y, Tang C, Rasmussen D (2012) A large-scale model of the functioning brain. Science 338(6111):1202-1205

Fountas Z, Shanahan M (2017) The role of cortical oscillations in a spiking neural network model of the basal ganglia. PloS One 12:e0189109

Gilbertson T, Steele D (2020) Tonic dopamine, uncertainty and basal ganglia action selection. Neuroscience 466:109-124

Girard B, Cuzin V, Guillot A, Gurney KN, Prescott TJ (2003) A basal ganglia inspired model of action selection evaluated in a robotic survival task. J Integr Neurosci 2(2):179-200

Girard B, Filliat D, Meyer JM, Berthoz A, Guillot A (2005) Integration of navigation and action selection functionalities in a computational model of cortico-basal-ganglia-thalamo-cortical loops. Adapt Behav 13:97-113

Girard B, Lienard J, Gutierrez CE, DeLord B, Doya K (2021) A biologically constrained spiking neural network model of the primate basal ganglia with overlapping pathways exhibits action selection. Eur J Neurosci 53:2254-2277
Girard B, Tabareau N, Pham QC, Berthoz A, Slotine JJ (2008) Where neuroscience and dynamic system theory meet autonomous robotics: a contracting basal ganglia model for action selection. Neural Netw 21:628-641

Gittis AH, Berke JD, Bevan MD, Chan CS, Mallet N, Morrow MM, Schmidt R (2014) New roles for the external globus pallidus in basal ganglia circuits and behavior. J Neurosci 34(46):1517815183

Graybiel AM (2000) The basal ganglia. Curr Biol 10:R509-R511

Gurney K, Prescott TJ, Redgrave P (2001) A computational model of action selection in the basal ganglia I: a new functional anatomy. Biol Cybern 85:401-410

Gurney K, Prescott TJ, Redgrave P (2001) A computational model of action selection in the basal ganglia II: analysis and simulation of behaviour. Biol Cybern 85:411-423

Gurney K, Prescott TJ, Wickens JR, Redgrave P (2004) Computational models of the basal ganglia: from robots to membranes. Trends Neurosci 27(8):453-459

Gurney KN, Humphries MD, Redgrave P (2015) A new framework for cortico-striatal plasticity: behavioural theory meets in vitro data at the reinforcement-action interface. PLoS Biol 13(1):e1002034

Hjorth JJJ, Kozlov A, Carannante I, Frost Nylén J, Lindroos R, Johansson Y, Tokarska A, Dorst MC, Suryanarayana SM, Silberberg G, Kotaleski JH, Grillner S (2020) The microcircuits of striatum in silico. Proc Natl Acad Sci USA 117:9554-9565

Humphries MD, Gurney K (2012) Network effects of subthalamic deep brain stimulation drive a unique mixture of responses in basal ganglia output. Eur J Neurosci 36:2240-2251

Humphries MD, Gurney KN (2002) The role of intra-thalamic and thalamocortical circuits in action selection. Netw Comput Neural Syst 13:131-156

Humphries MD, Prescott TJ (2010) The ventral basal ganglia, a selection mechanism at the crossroads of space, strategy, and reward. Prog Neurobiol 90:385-417

Humphries MD, Stewart RD, Gurney KN (2006) A physiologically plausible model of action selection and oscillatory activity in the basal ganglia. J Neurosci 26:12921-12942

Humphries MD, Wood R, Gurney K (2009) Dopamine-modulated dynamic cell assemblies generated by the gabaergic striatal microcircuit. Neural Netw 22:1174-1188

Humphries MD, Wood R, Gurney K (2010) Reconstructing the threedimensional gabaergic microcircuit of the striatum. PLoS Comput Biol 6:e1001011

Kawaguchi Y (1993) Physiological, morphological, and histochemical characterization of three classes of interneurons in rat neostriatum. J Neurosci 13(11):4908-4923

Khamassi M, Humphries MD (2012) Integrating cortico-limbic-basal ganglia architectures for learning model-based and model-free navigation strategies. Front Behav Neurosci 6:79

Khamassi M, Lacheze L, Girard B, Berthoz A, Guillot A (2005) Actorcritic models of reinforcement learning in the basal ganglia: from natural to artificial rats. Adapt Behav 13:131-148

Lepora NF, Gurney KN (2012) The basal ganglia optimize decision making over general perceptual hypotheses. Neural Comput 24(11):2924-2945

Lindahl M, Kotaleski JH (2016) Untangling basal ganglia network dynamics and function: role of dopamine depletion and inhibition investigated in a spiking network model. eNeuro. https://doi. org/10.1523/ENEURO.0156-16.2016

Liénard J, Girard B (2014) A biologically constrained model of the whole basal ganglia addressing the paradoxes of connections and selection. J Comput Neurosci 36:445

Mallet N, Micklem BR, Henny P, Brown MT, Williams C, Bolam JP, Nakamura KC, Magill PJ (2012) Dichotomous organization of the external globus pallidus. Neuron 74:1075-1086 
Marsden CD, Obeso JA (1994) The functions of the basal ganglia and the paradox of stereotaxic surgery in parkinson's disease. Brain 117(Pt 4):877-897

Mink JW (1996) The basal ganglia: focused selection and inhibition of competing motor programs. Prog Neurobiol 50:381-425

Mink JW, Thach WT (1993) Basal ganglia intrinsic circuits and their role in behavior. Curr Opin Neurobiol 3:950-957

Parent A, Hazrati LN (1995) Functional anatomy of the basal ganglia. II. The place of subthalamic nucleus and external pallidum in basal ganglia circuitry. Brain Res Brain Res Rev 20(1):128-154

Prescott TJ, Montes Gonzalez FM, Gurney K, Humphries MD, Redgrave P (2006) A robot model of the basal ganglia: behavior and intrinsic processing. Neural Netw 19:31-61

Prescott TJ, Redgrave P, Gurney K (1999) Layered control architectures in robots and vertebrates. Adapt Behav 7:99-127

Rajakumar N, Elisevich K, Flumerfelt BA (1994) The pallidostriatal projection in the rat: a recurrent inhibitory loop? Brain Res 651:332-336

Redgrave P, Costa R (2021) Chapter 38: the basal ganglia. In: Kandel E, Koester J, Mack S, Siegelbaum S (eds) Principles of neural science. McGraw-Hill, New York, pp 932-952

Redgrave P, Prescott TJ, Gurney K (1999) The basal ganglia: a vertebrate solution to the selection problem? Neuroscience 89(4):10091023

Spreizer S, Angelhuber M, Bahuguna J, Aertsen A, Kumar A (2017) Activity dynamics and signal representation in a striatal network model with distance-dependent connectivity. eNeuro. https://doi. org/10.1523/ENEURO.0348-16.2017

Stafford T, Gurney KN (2004) The role of response mechanisms in determining reaction time performance: Pieron's law revisited. Psychono Bull Rev 11:975-987

Stafford T, Gurney KN (2007) Biologically constrained action selection improves cognitive control in a model of the stroop task. Philosophical Transactions of the Royal Society of London. Ser B Biol Sci 362:1671-1684
Stewart TC, Bekolay T, Eliasmith C (2012) Learning to select actions with spiking neurons in the basal ganglia. Front Neurosci 6:2

Suryanarayana SM, Hellgren Kotaleski J, Grillner S, Gurney KN (2019) Roles for globus pallidus externa revealed in a computational model of action selection in the basal ganglia. Neural Net 109:113136

Tepper JM, Koós T, Ibanez-Sandoval O, Tecuapetla F, Faust TW, Assous M (2018) Heterogeneity and diversity of striatal gabaergic interneurons: update 2018. Front Neuroanat 12:91

Tomkins A, Vasilaki E, Beste C, Gurney K, Humphries M (2014) Transient and steady-state selection in the striatal microcircuit. Front Comput Neurosci 7:192

Walker RH, Arbuthnott GW, Wright AK (1989) Electrophysiological and anatomical observations concerning the pallidostriatal pathway in the rat. Exp Brain Res 74(2):303-310

Wilson CJ, Groves PM (1980) Fine structure and synaptic connections of the common spiny neuron of the rat neostriatum: a study employing intracellular inject of horseradish peroxidase. J Comp Neurol 194(3):599-615

Yartsev MM, Hanks TD, Yoon AM, Brody CD (2018) Causal contribution and dynamical encoding in the striatum during evidence accumulation. eLife 7:34929

Yim MY, Aertsen A, Kumar A (2011) Significance of input correlations in striatal function. PLoS Comput Biol 7(11):e1002254

Zhang J, Bogacz R (2010) Optimal decision making on the basis of evidence represented in spike trains. Neural Comput 22(5):11131148

Publisher's Note Springer Nature remains neutral with regard to jurisdictional claims in published maps and institutional affiliations. 